PROCEEDINGS OF THE

AMERICAN MATHEMATICAL SOCIETY

Volume 139, Number 2, February 2011, Pages 385-389

S 0002-9939(2010)10625-1

Article electronically published on September 27, 2010

\title{
ON THE UPPER CENTRAL SERIES OF INFINITE GROUPS
}

\author{
M. DE FALCO, F. DE GIOVANNI, C. MUSELLA, AND Y. P. SYSAK
}

(Communicated by Jonathan I. Hall)

To Bernhard Amberg on his 70th birthday

\begin{abstract}
Two relevant theorems by R. Baer and P. Hall show that a group is finite over a term with finite ordinal type of its upper central series if and only if it is finite-by-nilpotent. Extending these results, we prove here that if $G$ is any group, the hypercentre factor group $G / \bar{Z}(G)$ is finite if and only if $G$ contains a finite normal subgroup $N$ such that $G / N$ is hypercentral (where the hypercentre $\bar{Z}(G)$ of $G$ is defined as the last term of its upper central series).
\end{abstract}

\section{INTRODUCTION}

It was proved by R. Baer 2 that if a term $Z_{n}(G)$ of the upper central series of a group $G$ has finite index in $G$ for some positive integer $n$, then the term $\gamma_{n+1}(G)$ of the lower central series of $G$ is finite (see also [6], Part 1, p. 113). Of course, this result is a generalization of the celebrated theorem by I. Schur [7] on the finiteness of the commutator subgroup of a group which is finite over its centre. It turns out immediately that Baer's theorem cannot be extended to terms with infinite ordinal type of the upper and lower central series of a group. In fact, the locally dihedral 2-group $D\left(2^{\infty}\right)$ is hypercentral, but its hypocentre is the subgroup of type $2^{\infty}$; in particular, it can occur that the hypercentre of a group has finite index, while its hypocentre is infinite. Recall here that the hypercentre $\bar{Z}(G)$ of a group $G$ is the last term of the upper central series of $G$, and $G$ is hypercentral if it coincides with its hypercentre; similarly, the hypocentre $\bar{\gamma}(G)$ of $G$ is the last term of its lower central series, and $G$ is hypocentral if $\bar{\gamma}(G)=\{1\}$. Observe that, although the properties of being hypercentral, hypocentral or nilpotent coincide for a finite group, in general the class of hypercentral groups and that of hypocentral groups behave quite differently; for instance, every hypercentral group is locally nilpotent, while all free groups are hypocentral.

The consideration of infinite extraspecial groups shows that the converse of Baer's theorem does not hold in general; however, P. Hall 3 proved that if $G$ is a group such that $\gamma_{n+1}(G)$ is finite for some positive integer $n$, then the in$\operatorname{dex}\left|G: Z_{2 n}(G)\right|$ is likewise finite. Thus the theorems of Baer and Hall together essentially state that a group is finite over a term with finite ordinal type of its upper central series if and only if it is finite-by-nilpotent. Our main result shows

Received by the editors November 6, 2009.

2010 Mathematics Subject Classification. Primary 20E15.

Key words and phrases. Upper central series, hypercentre, hypercentral group.

(C)2010 American Mathematical Society Reverts to public domain 28 years from publication 
that, replacing nilpotency by hypercentrality this remains true also for terms with arbitrary ordinal type of the upper central series.

Theorem. Let $G$ be a group. The factor group $G / \bar{Z}(G)$ is finite if and only if there exists a finite normal subgroup $N$ of $G$ such that $G / N$ is hypercentral.

In the above statement it cannot be proved that if the index $\left|G: Z_{\alpha}(G)\right|$ is finite for some ordinal $\alpha$, then $G$ contains a finite normal subgroup $N$ such that $G / N$ is hypercentral with length at most $\alpha$; in fact, in the locally dihedral 2 -group we have that $Z_{\omega}\left(D\left(2^{\infty}\right)\right)$ has index 2 in $D\left(2^{\infty}\right)$, but all infinite factor groups of $D\left(2^{\infty}\right)$ have hypercentral length $\omega+1$. Observe however that if $G / Z_{\alpha}(G)$ is finite and $\bar{\gamma}\left(G / Z_{\alpha}(G)\right)=\gamma_{k+1}\left(G / Z_{\alpha}(G)\right)$, then every hypercentral homomorphic image of $G$ has length at most $\alpha+k$. Finally, note that if $F$ is a free non-abelian group, then $\gamma_{\omega}(F)=Z(F)=\{1\}$ and hence P. Hall's theorem cannot be extended to terms with infinite ordinal type of the lower central series of a group.

Most of our notation is standard and can be found in [6].

\section{Proof of THE THEOREM}

Our first lemma deals with the case of torsion-free groups.

Lemma 1. Let $G$ be a torsion-free group such that $G / Z_{\alpha}(G)$ is locally finite for some ordinal $\alpha$. Then $G=Z_{\alpha}(G)$.

Proof. Let $E$ be any finitely generated subgroup of $G$. As $E / E \cap Z_{\alpha}(G)$ is finite, the subgroup $E \cap Z_{\alpha}(G)$ is finitely generated and hence it is nilpotent. Thus $E$ satisfies the maximal condition on subgroups, and so $E \cap Z_{\alpha}(G)$ is contained in $Z_{n}(E)$ for some positive integer $n$. In particular, $E / Z_{n}(E)$ is finite and it follows from Baer's theorem that $\gamma_{n+1}(E)$ is also finite. Therefore $\gamma_{n+1}(E)=\{1\}$ since $G$ is torsion-free, so that $G$ is locally nilpotent. P. Hall's theory of isolators now yields that $G / Z_{\alpha}(G)$ must be torsion-free (see [5], 2.3.9(i)), so that $G=Z_{\alpha}(G)$ and the statement is proved.

It follows easily from Baer's theorem that if the factor group $G / Z_{n}(G)$ is locally finite for some positive integer $n$, then the subgroup $\gamma_{n+1}(G)$ is likewise locally finite and so $G$ is (locally finite)-by-(nilpotent of class at most $n$ ). Our next result extends this property to arbitrary ordinal types.

Lemma 2. Let $G$ be a group such that $G / Z_{\alpha}(G)$ is locally finite for some ordinal $\alpha$. Then there exists a locally finite normal subgroup $N$ of $G$ such that $G / N$ is hypercentral with length (at most) $\alpha$.

Proof. Without loss of generality, it can be assumed that $G$ has no periodic nontrivial normal subgroups, so that in particular $Z_{\alpha}(G)$ is torsion-free. Let $x$ be any element of finite order of $G$. Then $\langle x\rangle$ is an ascendant subgroup of $\left\langle x, Z_{\alpha}(G)\right\rangle$, so that

$$
\left\langle x, Z_{\alpha}(G)\right\rangle=\langle x\rangle \times Z_{\alpha}(G)
$$

and $\left[Z_{\alpha}(G), x\right]=\{1\}$. Let $K$ be the subgroup generated by all elements of finite order of $G$. Then $\left[Z_{\alpha}(G), K\right]=\{1\}$ and hence $K \cap Z_{\alpha}(G)$ is contained in $Z(K)$. It follows that $K / Z(K)$ is locally finite, so that $K^{\prime}$ is locally finite by Schur's theorem. Thus $K$ itself is periodic and hence $K=\{1\}$. Therefore $G$ is torsion-free and so $G=Z_{\alpha}(G)$ by Lemma 1 . The statement is proved. 
Lemma 3. Let $G$ be a group, and let $N$ be a finite non-trivial normal subgroup of $G$ such that $G / N$ is hypercentral and $[N, G]=N$. Then there exists a subgroup $K$ of $G$ such that $G=K N$ and $K \cap N<N$.

Proof. By Zorn's Lemma there exists a normal subgroup $M$ of $G$ which is maximal with respect to the condition that $N$ is not contained in $M$. Write $\bar{G}=G / M$. Then $\bar{N}$ is contained in every non-trivial normal subgroup of $\bar{G}$, and in particular $\bar{N}$ is the unique minimal normal subgroup of $\bar{G}$. If $\bar{N}$ is abelian, as $[\bar{N}, \bar{G}]=\bar{N}$ and $\bar{G} / \bar{N}$ is hypercentral, it is well known that $\bar{G}$ splits over $\bar{N}$ (see for instance 1], Theorem 5.3.11), and hence there exists a subgroup $K$ of $G$ such that $G=K N$ and

$$
K \cap N \leq M \cap N<N .
$$

Suppose that $\bar{N}$ is not abelian, and let $\bar{P}$ be a non-trivial Sylow subgroup of $\bar{N}$, so that $\bar{G}=N_{\bar{G}}(\bar{P}) \bar{N}$ by Frattini's argument; in this case put $\bar{K}=N_{\bar{G}}(\bar{P})$. Then $G=K N$ and $K \cap N$ is properly contained in $N$, since

$$
\bar{K} \cap \bar{N}=N_{\bar{N}}(\bar{P})<\bar{N} .
$$

The lemma is proved.

Proof of the Theorem. Suppose first that $G / \bar{Z}(G)$ is finite. Assume for a contradiction that the statement is false, and choose a counterexample $G$ for which $G / \bar{Z}(G)$ has smallest order $m$. Put $Z=\bar{Z}(G)$, and let $M$ be any maximal subgroup of $G$ containing $Z$. As $Z$ is contained in the hypercentre of $M$, by the minimal choice of $m$ we have that $M$ contains a finite normal subgroup $K$ such that $M / K$ is hypercentral. Clearly, $K$ has finitely many conjugates in $G$, so that its normal closure $K^{G}$ is finite and in particular $G / K^{G}$ is not hypercentral; on the other hand, $Z K^{G} / K^{G}$ lies in the hypercentre of $G / K^{G}$, so that $Z K^{G} / K^{G}$ has index $m$ in $G / K^{G}$ and hence $K^{G}$ is contained in $Z$. Therefore $M / Z$ is nilpotent and so all proper subgroups of $G / Z$ are nilpotent. Since the finite group $G / Z$ has trivial centre, it follows from a well known result of O.J. Šmidt that the group $G / Z$ is the semidirect product of a normal subgroup $A / Z$ by a cyclic subgroup $\langle b Z\rangle$, where $A / Z$ is a minimal normal subgroup of $G / Z$ having prime exponent $p$ and the coset $b Z$ has prime order $q \neq p$ (see for instance [4, Satz 5.2).

As $A / Z$ is abelian, the subgroup $A$ is hypercentral, so that the elements of finite order of $A$ form a characteristic subgroup $C$, and $C=A \cap T$, where $T$ is the largest periodic normal subgroup of $G$. It follows from Lemma 2 that $G / C$ is (locally finite)-by-hypercentral; as $A / C$ is torsion-free and $|G / A|=q$, we have that $G / C$ is hypercentral, so that $C$ cannot be contained in $Z$ and hence $A=Z C$. Thus $A=Z U$, where $U$ is a finite subgroup of $C$. Since all hypercentral groups are locally nilpotent (see for instance [6, Part 1, p. 50), the subgroup $\langle U, b\rangle$ is polycyclic-by-finite, and so the normal closure $U^{\langle b\rangle}$ is finite. Since $A=Z U^{\langle b\rangle}$, we may choose a finite $\langle b\rangle$-invariant subgroup $V$ with smallest order with respect to the condition $A=Z V$. The $\langle b\rangle$-invariant subgroup $[V,\langle b\rangle]$ obviously is contained in $V$ but not in $Z$. Moreover, $\langle b\rangle$ acts irreducibly on $A / Z$ and so also on $V / V \cap Z$, and hence

$$
V=[V,\langle b\rangle](V \cap Z)
$$


Thus $A=Z V=Z[V,\langle b\rangle]$, and the assumption on the order of $V$ yields that $[V,\langle b\rangle]=V$. Let $E$ be any finitely generated subgroup of $G$ containing $\langle V, b\rangle$, and assume that $\left[V, Z_{n}(E)\right] \neq\{1\}$ for some positive integer $n$; consider the smallest positive integer $r$ with such a property. Clearly, there is a positive integer $s<r$ such that $\left[V, Z_{r}(E)\right]$ is contained in $Z_{s}(E)$ but not in $Z_{s-1}(E)$. Then

$$
\left[V, Z_{r}(E),\langle b\rangle\right] \leq\left[Z_{s}(E),\langle b\rangle\right] \leq Z_{s-1}(E)
$$

and

$$
\left[Z_{r}(E),\langle b\rangle, V\right] \leq\left[Z_{r-1}(E), V\right]=\{1\},
$$

so that $\left[\langle b\rangle, V, Z_{r}(E)\right]$ is contained in $Z_{s-1}(E)$ by the Three Subgroup Lemma, a contradiction because $[V,\langle b\rangle]=V$. Therefore $\left[V, Z_{n}(E)\right]=\{1\}$, for all positive integers $n$. Since $E$ is polycyclic-by-finite, we have that $E \cap Z$ lies in $Z_{n}(E)$ for some $n$, and so $[V, E \cap Z]=\{1\}$. It follows that $[V, Z]=\{1\}$, and hence $V$ is a normal subgroup of

$$
G=\langle A, b\rangle=\langle Z, V, b\rangle
$$

On the other hand, the subgroup $\langle Z, b\rangle$ is obviously hypercentral, so that $G / V$ is likewise hypercentral and $G$ is finite-by-hypercentral. This contradiction shows that $G$ contains a finite normal subgroup $L$ such that $G / L$ is hypercentral.

Assume that the converse statement is false, and choose a counterexample $G$ for which the finite normal subgroup $N$ such that $G / N$ is hypercentral has smallest possible order. Clearly, the factor group $G / \bar{Z}(G)$ is likewise a minimal counterexample, and hence replacing $G$ by $G / \bar{Z}(G)$ we may suppose without loss of generality that $Z(G)=\{1\}$. Moreover, as $G /[N, G]$ is hypercentral, we have that $[N, G]=N$. By Lemma 3 we have that $G$ contains a subgroup $K$ such that $G=K N$ and $K \cap N<N$. As $K / K \cap N$ is hypercentral, by the minimal choice of $G$ we have that the hypercentre $H=\bar{Z}(K)$ of $K$ has finite index in $K$ and so also in $G$. Then the subgroup

$$
C_{H}(N)=\bar{Z}(K) \cap C_{G}(N)
$$

is infinite, and hence $Z(K) \cap C_{G}(N) \neq\{1\}$. On the other hand, $Z(K) \cap C_{G}(N)$ is contained in the centre of $G$, and this contradiction completes the proof of the theorem.

\section{REFERENCES}

[1] B. Amberg, S. Franciosi and F. de Giovanni, Products of Groups, Clarendon Press, Oxford, 1992. MR.1211633 (94h:20001)

[2] R. Baer, Endlichkeitskriterien für Kommutatorgruppen, vol. 124, Math. Ann., 1952, pp. 161177. MR0045720(13:622a)

[3] P. Hall, Finite-by-nilpotent groups, vol. 52, Proc. Cambridge Philos. Soc., 1956, pp. 611-616. MR0080095 (18:190d)

[4] B. Huppert, Endliche Gruppen I, Springer, Berlin, 1983. MR0224703 (37:302)

[5] J.C. Lennox and D.J.S. Robinson, Infinite Soluble Groups, Oxford University Press, Oxford, 2004. MR2093872 (2006b:20047)

[6] D.J.S. Robinson, Finiteness Conditions and Generalized Soluble Groups, Springer, Berlin, 1972. MR0332989 (48:11314) MR0332990 (48:11315)

[7] I. Schur, Neuer Beweis eines Satzes über endliche Gruppen, Proc. Sympos. Appl. Math., Sitzber. Akad. Wiss., Berlin, 1902, pp. 1013-1019. 
Dipartimento di Matematica e Applicazioni, Università di Napoli Federico II, Complesso Universitario Monte S. Angelo, Via Cintia, I-80126 Napoli, Italy

E-mail address: mdefalco@unina.it

Dipartimento di Matematica e Applicazioni, Università di Napoli Federico II, Complesso Universitario Monte S. Angelo, Via Cintia, I-80126 Napoli, Italy

E-mail address: degiovan@unina.it

Dipartimento di Matematica e Applicazioni, Università di Napoli Federico II, Complesso Universitario Monte S. Angelo, Via Cintia, I-80126 Napoli, Italy

E-mail address: cmusella@unina.it

Institute of Mathematics, Ukrainian National Academy of Sciences, vul. TereshChenkivska 3, $01601 \mathrm{KIEV}$, Ukraine

E-mail address: sysak@imath.kiev.ua 